\title{
Reducing loss to follow-up during treatment for drug-resistant tuberculosis
}

\author{
Ibrahim Abubakar ${ }^{1,2}$ and Marc Lipman $2,3,4$
}

Affiliations: ${ }^{1}$ Institute for Global Health, University College London, London, UK. ${ }^{2}$ UCL-TB, University College London, London, UK. ${ }^{3} \mathrm{UCL}$ Respiratory, Division of Medicine, University College London, London, UK. ${ }^{4}$ Royal Free London NHS Foundation Trust, London, UK.

Correspondence: Marc Lipman, Royal Free London NHS Foundation Trust, Respiratory Medicine, Pond Street, London, NW3 2QG, UK. E-mail: marclipmanखिhs.net

@ERSpublications

Loss to follow-up during treatment for drug-resistant TB can be reduced by regular and sustained psychosocial support provided by the patient's healthcare workers http://ow.ly/VzkI30n4sni

Cite this article as: Abubakar I, Lipman M. Reducing loss to follow-up during treatment for drug-resistant tuberculosis. Eur Respir J 2019; 53: 1802268 [https://doi.org/10.1183/13993003.02268-2018].

The global strategy to end tuberculosis (TB) highlights integrated patient-centred care, bold policies and supportive systems, and intensified research and innovation as major priorities [1]. Central to this is the need to keep people in care and ensure that they are successfully treated using the best evidence-based approaches. This is of particular relevance to people with drug-resistant disease, who will generally need treatment for longer and require a more complicated, potentially toxic regimen. In this issue of the European Respiratory Journal, LAW et al. [2] summarise, in their systematic literature review, the evidence for measures that minimise loss to follow-up during treatment of drug-resistant TB in adults.

The synthesis of results suggests that supplementing standard care with psychosocial, educational or material support decreased loss to follow-up (defined as treatment interruption of two or more months). The greatest effect was seen when daily home visits were combined with directly observed therapy (DOT).

The pooled analysis included 5114 participants from 31 studies, almost all of which were from high TB or high multidrug-resistant TB burden areas, and further supported the observation that frequent contact between healthcare workers and patients using direct observation and home visits, especially when sustained throughout treatment, was associated with better outcomes (lower loss to follow-up). Furthermore, financial incentives such as travel or wage reimbursement were also associated with less loss to follow-up than found in comparator groups. However, the high level of heterogeneity in the overall pooled and sub-group analyses (in part explained by the use of different controls in each study) suggests that these results need to be interpreted carefully.

The authors were able to distinguish the effect of psychosocial support from that due to direct observation of treatment itself. When DOT was undertaken at least daily, less loss to follow-up occurred in participants who received individual support throughout treatment compared to those having fixed or unspecified support. The systematic review, however, failed to detect a significant effect from group support, that involving a patient's family, or when nutritional advice and supplementation was provided.

The review focused on cohort studies and quasi-experimental designs and, appropriately, did not exclude any studies based on their assessment of quality. Only two cluster trials were included, which both lacked masking of participants. As with most reviews of observational data, the authors acknowledge a range of limitations inherent within the included studies, such as differences in the two arms due to "before and 
after" designs, missingness of data, inadequate description of interventions, plus lack of adjustment for confounding. Furthermore, limited reporting affected the ability to assess the fidelity of interventions.

Despite their best efforts (which included contacting the authors of the original studies), the researchers were often unable to determine what it was in the "counselling" offered to patients that led to success. As indicated above, an important element appeared to be the one-on-one time spent between patients and healthcare workers that addressed psychological, social or treatment-related issues. Given the variation in what this meant in practice, including the differences in training provided to healthcare workers across studies, it is tempting to speculate that reduced loss to follow-up may be a consequence of the patient perceiving someone has a sustained (and positive) interest in them whilst they undergo treatment.

The exclusion of participants who died or were "transferred out" and did not have a final outcome reported, as well as those for whom treatment failed, is a potential source of bias. These events may have been triggered by non-adherence and therefore such individuals could potentially be systematically more likely to have been "lost to follow-up". However, sensitivity analyses found that categorising those who transferred out or died as lost to follow-up produced similar results.

Adherence, as measured by the successful intake of pills by patients, determines in large part treatment response. Unfortunately, too few studies reported adherence outcomes. We welcome the careful analysis presented that considers the added value of adherence-supporting measures and incentives (financial or nutritional) and psychosocial support over and above direct observation, which has been central to many TB programmes. However, adherence, and most probably consequent prevention of loss to follow-up, can also be supported through other approaches that enable patients to take their medication and remain in care. Novel interventions evaluated recently in drug-sensitive TB include video observed therapy [3] and electronic medication monitors [4]. Structural and system support measures, such as the provision of accommodation for multidrug-resistant $\mathrm{TB}$ patients (e.g. a hostel within a community rather than a more isolated sanatorium) require formal evaluation.

Ultimately, loss to follow-up and the ability to take medication as prescribed (adherence) is a reflection of individual human behaviour. Improvements in outcome require an appreciation of the behaviour models than underpin human action such as the "perceptions and practicalities approach" [5], which argues that adherence is influenced by a combination of perceptual factors (e.g. beliefs about the illness and treatment) and practical factors (e.g. capability and resources linked to individual and socioeconomic elements) that affect an individual's motivation and ability to start and continue with treatment (figure 1). People differ in the key drivers that determine whether they respond to an incentive or psychological support. These are

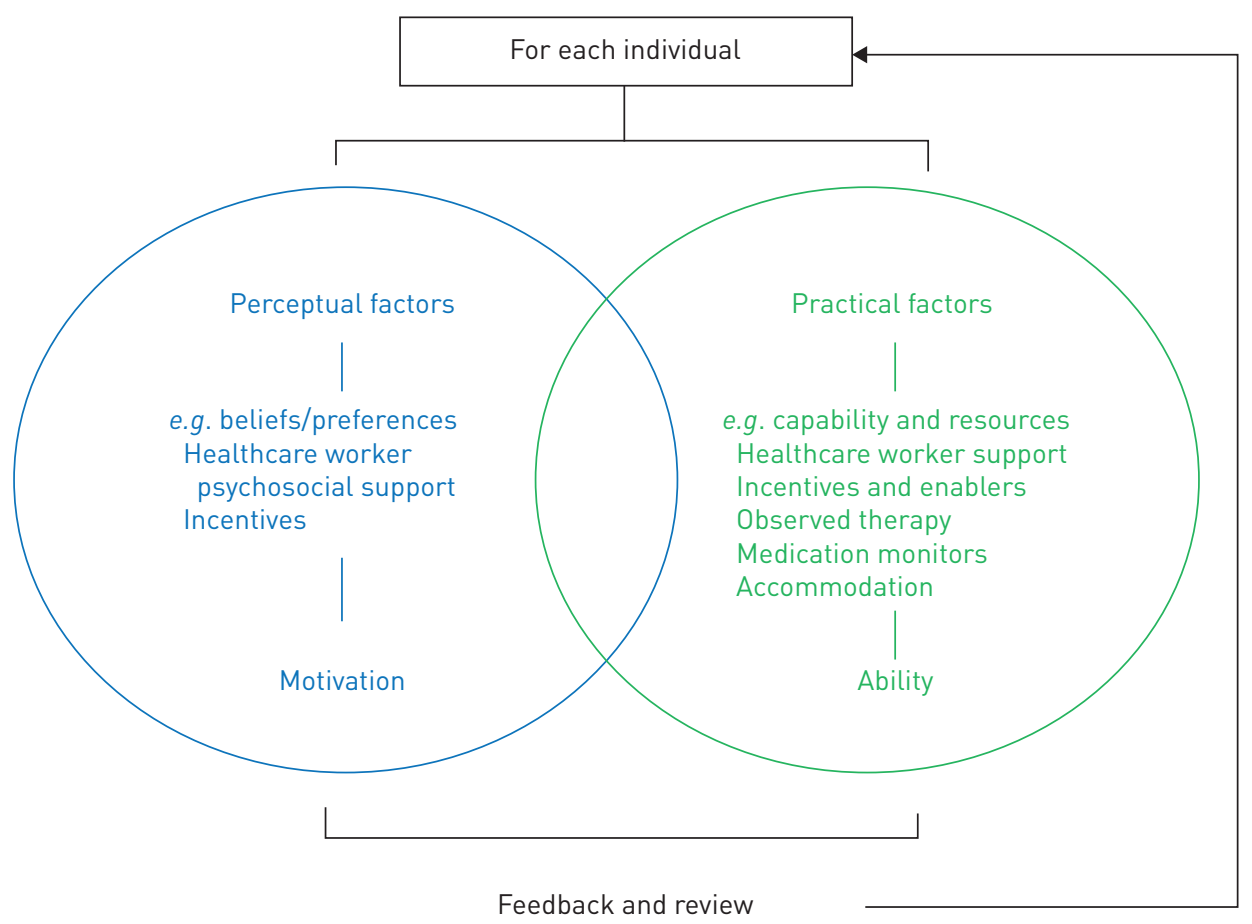

FIGURE 1 Perceptions and practicalities model. Adapted from [5]. 
more likely to be effective when tailored to the needs of that person; and this can be achieved using a menu-based approach to address both perceptual and practical barriers to adherence [6]. This partly explains the modest effect size of most interventions designed to maximise treatment success, as they may not mitigate the many drivers of non-adherence and loss to follow-up that may be present in a single individual. TB needs to learn from areas of medicine such as HIV medicine [7], where loss to follow-up through the cascade of care is recognised as a major issue, and much effort is expended to try to minimise this [8]. Behavioural science is seen to have a role to address adherence, and sensibly evaluate novel support measures [6].

Within the wider literature, there is an understanding that to achieve good adherence and reduce loss to follow-up, health systems need to consider pragmatic approaches, such as the use of a manualised intervention that enables both a rapid assessment of an individual's need and the introduction of the relevant support measure most likely to ensure best outcome [9]. However, the use of manualised interventions is likely to be resource-intensive, and may not be feasible in low and middle income countries.

A determinant of the subsequent value of interventions on clinical outcomes is the degree by which it is possible to implement such measures in routine practice. In this regard the authors identified a number of challenges that arose within the reported studies. These ranged from healthcare worker reluctance to use new measures, to inadequate training, and difficulty in implementing the specific intervention.

There is much to be learnt from the results of this paper, and it will be important to see whether the findings are replicated as treatment for drug-resistant TB moves away from a "core" injectable agent to possibly better-tolerated oral therapies [10]. This has considerable operational advantages, as does the use of self-administered therapy. The merits of combining this with interventions that can achieve good adherence is obvious, though the evidence base to support this approach is currently weak. We agree with the authors' call for randomised controlled trials assessing patient, healthcare worker and system approaches that will provide the evidence needed to ensure adherence and minimise loss to follow-up. The global effort to end TB depends on this.

Acknowledgements: M. Lipman is Chief Investigator on the NIHR (National Institute for Health Research, England) Health Technology Assessment-funded study "Intervening with a Manualised Package to AChieve treatment adherence in people with Tuberculosis: the IMPACT study" (16/88/06). The views expressed are those of the authors and not necessarily those of the NHS (National Health Service, UK), the NIHR or the Department of Health and Social Care.

Conflict of interest: I. Abubakar has nothing to disclose. M. Lipman reports being awarded an NIHR HTA grant for the IMPACT study to improve adherence in people taking treatment for TB.

\section{References}

1 World Health Organization. WHO End TB Strategy. Geneva, WHO, 2015. Available from: www.who.int/tb/ post2015_strategy/en/

2 Law C, Daftary A, O’Donnell M, et al. Interventions to improve retention-in-care and treatment adherence among patients with drug-resistant tuberculosis: a systematic review. Eur Respir J 2019; 53: 1801030.

3 Story A, Aldridge RW, Smith CM, et al. Smartphone-enabled video observed versus directly observed treatment for tuberculosis: a randomised controlled trial. Lancet 2018; in press.

4 Liu X, Lewis JJ, Zhang $\mathrm{H}$, et al. Effectiveness of electronic reminders to improve medication adherence in tuberculosis patients: a cluster-randomised trial. PLoS Med 2015; 12: e1001876.

5 Horne R, Weinman J, Barber N, et al. Concordance, adherence and compliance in medicine taking. London, National Co-ordinating Centre for NHS Service Delivery and Organisation R\&D, 2006. Available from: www. netscc.ac.uk/hsdr/files/project/SDO_FR_08-1412-076_V01.pdf

6 National Institute for Health and Care Excellence. Medicines Adherence: Involving Patients in Decisions about Prescribed Medicines and Supporting Adherence. Guidance and Guidelines. London, NICE, 2016. Available from: www.nice.org.uk/guidance/cg76

7 Horne R, Cooper V, Gellaitry G, et al. Patients' perceptions of highly active antiretroviral therapy in relation to treatment uptake and adherence: the utility of the necessity-concerns framework. J Acquir Immune Defic Syndr 2007; 45: 334-341.

8 Levi J, Raymond A, Pozniak A, et al. Can the UNAIDS 90-90-90 target be achieved? A systematic analysis of national HIV treatment cascades. BMJ Glob Health 2016; 1: e000010.

9 National Institute for Health Research. Journals Library. Intervening with a Manualised Package to Achieve Treatment Adherence in People with Tuberculosis: the IMPACT study. www.journalslibrary.nihr.ac.uk/ programmes/hta/168806/\#/ Date last updated: July 21, 2016. Date last accessed: November 20, 2018.

10 World Health Organization. Rapid Communication: Key Changes to Treatment of Multidrug- and Rifampicin-resistant Tuberculosis (MDR/RR-TB). Geneva, WHO, 2018. Available from: www.who.int/tb/ publications/2018/WHO_RapidCommunicationMDRTB.pdf?ua=1 